\title{
A conceptual ENSO model under realistic noise forcing
}

\author{
J. Saynisch, J. Kurths, and D. Maraun \\ Nonlinear Dynamics Group, University Potsdam, Germany \\ Received: 16 January 2006 - Revised: 20 April 2006 - Accepted: 26 April 2006 - Published: 13 July 2006
}

\begin{abstract}
We investigated the influence of atmospheric noise on the generation of interannual El Niño variability. Therefore, we perturbed a conceptual ENSO delay model with surrogate windstress data generated from tropical windspeed measurements. The effect of the additional stochastic forcing was studied for various parameter sets including periodic and chaotic regimes. The evaluation was based on a spectrum and amplitude-period relation comparison between model and measured sea surface temperature data. The additional forcing turned out to increase the variability of the model output in general. The noise-free model was unable to reproduce the observed spectral bandwidth for any choice of parameters. On the contrary, the stochastically forced model is capable of producing a realistic spectrum. The weakly nonlinear regimes of the model exhibit a proportional relation between amplitude and period matching the relation derived from measurement data. The chaotic regime, however, shows an inversely proportional relation. A stability analysis of the different regimes revealed that the spectra of the weakly nonlinear regimes are robust against slight parameter changes representing disregarded physical mechanisms, whereas the chaotic regime exhibits a very unstable realistic spectrum. We conclude that the model including stochastic forcing in a parameter range of moderate nonlinearity best matches the real conditions. This suggests that atmospheric noise plays an important role in the coupled tropical pacific ocean-atmosphere system.
\end{abstract}

\section{Introduction}

Over thirty years ago Bjerknes (1969) described the basic feedback mechanisms underlying El Niño/Southern Oscilla-

Correspondence to: J. Saynisch

(saynisch@agnld.uni-potsdam.de) tion (ENSO). These mechanisms are based on coupling between the ocean and the atmosphere.

The observed interannual variability of ENSO can in principle be explained by deterministic dynamics: Strong nonlinear interactions between ocean and atmosphere lead to low order deterministic chaos (Timmermann and Jin, 2003). Nonlinear interactions of ENSO modes with the seasonal cycle can also lead to deterministic chaos (Tziperman, 1994, 1995). The latter concept is able to explain the locking of El Niño to the seasonal cycle.

Recently, a discussion has arisen about the influence of atmospheric noise on the ocean and the coupled oceanatmosphere system (Thompson and Battisti, 2001; Kessler, 2002; Fedorov, 2003). Models based solely on chaotic behavior cannot explain the full amount of ENSO variability, e.g. all models failed to predict the devastating 1997/98 El Niño (Fedorov, 2003). For this event, Lengaigne (2004) studied the effect of measured high- and low-frequency winds, based on GCM simulations. These investigations revealed that tropical wind anomalies (especially westerly wind bursts) are well able to influence the onset and growth of El Niños and even trigger a warm event. More conceptual examinations of this question consist of driving simple models with Gaussian noise (Stone and Price, 1998). Here the models are easily influenced by noise and are able to show stochastic resonance.

However, it is still under debate as to whether wind bursts are capable of causing ENSO variability or rather are the effect of the latter. Also, there is disagreement about whether typical atmospheric noise may at all be strong enough to influence the onset, progression, strength and period of $\mathrm{El}$ Niños.

Our approach to this controversial discussion is on a level of complexity between time consuming GCM studies and overly simple conceptual models disregarding essential physical mechanisms. We investigate the possible effects of - undoubtedly present - atmospheric noise, by utilizing

Published by Copernicus GmbH on behalf of the European Geosciences Union and the American Geophysical Union. 
Table 1. Parameter list of the GT model.

\begin{tabular}{cl}
\hline Parameter & Description \\
\hline$a, b$ & scaling parameters of additional noise drive \\
$A_{\text {Wind }}$ & equatorial additional noise drive \\
$A_{\text {Wind }_{\mathrm{n}}}$ & northern additional noise drive \\
$A^{*}$ & relates non-equatorial windstress to equatorial SST \\
$b_{0}$ & mean ocean-atmosphere coupling strength \\
$C_{o}$ & Kelvin wave propagation speed \\
$\tau_{\text {wind }}$ & wind affected fraction of basin crossing time \\
$\varepsilon$ & strength of seasonal variation \\
$\epsilon_{m}$ & damping coefficient of the ocean \\
$\epsilon_{T}$ & thermal damping coefficient \\
$h$ & thermocline depth anomaly \\
$H_{1}$ & upwelling layer depth \\
$\mu$ & seasonal varying coupling \\
$\rho$ & density of ocean water \\
$r_{e} / w$ & coastal boundary wave reflection coefficients \\
$\tau_{1,2}$ & basin crossing time ${ }_{1}$ Rossby, 2 Kelvin) \\
$T$ & temperature anomaly at eastern boundary \\
$T_{\text {sub }}$ & temperature anomaly at depth $H_{1}$ \\
$\bar{\omega}$ & mean upwelling \\
\hline
\end{tabular}

a conceptual model of considerable complexity and adding realistic stochastic forcing to the internal (i.e. deterministic) windstress. For this purpose we construct surrogates from measured windspeed data, in such a way that the low frequency variability is conserved while the high frequency part is randomized. This allows us to investigate the influence of realistic windstress on the spectrum, strength and period of ENSO on a conceptual basis. These investigations include chaotic and quasiperiodic model regimes.

The criteria to evaluate the quality of this stochastic extension are also based on measured data. We calculate spectra and amplitude-period relations (APR) of the model runs with the additional forcing and compare them with the same diagnostic measures from the NINO3 region of the Kaplan (1998) reconstruction and noise free model runs, respectively. Taking into account the natural origin of most model parameters, we performed a stability analysis. The model has to reproduce its spectrum and APR in a reasonable parameter space. A measure of robustness was introduced for this purpose.

The paper is organized as follows: In the second section the model and our stochastic forcing are introduced in more detail. The influence of the additional forcing is presented in Sect. 3. The last section presents our conclusions.

\section{Model and methodology}

\subsection{The model}

The investigations within this paper are based on the ENSO model of Galanti and Tziperman (2000), hereafter GT. This delay oscillator consists of two zonal ocean stripes and describes the eastern thermocline depth anomaly $h$ as a function of wind-excited Kelvin and Rossby waves (with parameters listed in Table 1):

$$
\begin{aligned}
h(t)= & e^{-\epsilon_{m}\left(\tau_{1}+\tau_{2}\right)} r_{w} r_{e} h\left(t-\tau_{1}-\tau_{2}\right) \\
& -e^{-\epsilon_{m}\left(\tau_{2}+\frac{\tau_{1}}{2}\right)} r_{w} \frac{A^{*}}{\beta \rho} \tau_{\text {Wind }} \tau_{1} \mu\left(t-\tau_{2}-\frac{\tau_{1}}{2}\right) \\
& * b_{0} T\left(t-\tau_{2}-\frac{\tau_{1}}{2}\right) \\
& +e^{-\epsilon_{m} \frac{\tau_{2}}{2}} \frac{\tau_{\text {Wind }} \tau_{2}}{\rho C_{o}} \mu\left(t-\frac{\tau_{2}}{2}\right) b_{0} T\left(t-\frac{\tau_{2}}{2}\right) .
\end{aligned}
$$

A change of zonal, equatorial windstress over the central $\mathrm{Pa}$ cific simultaneously generates upwelling (respectively downwelling) Kelvin waves and downwelling (respectively upwelling) Rossby waves (see Eq. 1). These waves travel to and from the Pacific ocean and are reflected at the coastal boundaries. In this way, they carry the upwelling and downwelling signal to the Ecuadorean coast and determine the eastern tropical pacific thermocline depth. Since the wave reflection and propagation weakens and delays the traveling signal, a nonintuitive and complex interplay of forces arises. For a detailed description of the model, see Galanti and Tziperman (2000).

The strength of the zonal windstress $\left(\tau_{x}\right)$ is assumed to be in balance with the eastern sea surface temperature (SST) and the ocean-atmosphere coupling (see Eq. 2). The latter varies according to the seasonal cycle $(\mu)$ around a mean value $\left(b_{0}\right)$ :

$$
\begin{aligned}
\tau_{x} & =b_{0} \mu T \\
\mu & =1+\varepsilon \cos (2 \pi t / 12-5 \pi / 6) .
\end{aligned}
$$

The SST at the eastern boundary is given as a function of thermal damping $\left(\epsilon_{T}\right)$ and upwelling $(\bar{\omega})$ :

$\partial_{t} T=-\epsilon_{T} T-\gamma \frac{\bar{\omega}}{H_{1}}\left(T-T_{\text {sub }}(h)\right)$

The temperature of the upwelling water is modeled as the hyperbolic tangent of the thermocline depth anomaly and represents the main nonlinearity in the GT (Galanti and Tziperman, 2000).

\subsection{Stochastic forcing}

To implement stochastic forcing, we extended the model with two additive terms: $A_{\text {Wind }_{n}}$ and $A_{\text {Winde }_{\mathrm{e}}}$. These terms were included in the part of the model that relates windstress to SST and thus represent additional windstress:

$$
\begin{aligned}
h(t)= & e^{-\epsilon_{m}\left(\tau_{1}+\tau_{2}\right)} r_{w} r_{e} h\left(t-\tau_{1}-\tau_{2}\right) \\
& -e^{-\epsilon_{m}\left(\tau_{2}+\frac{\tau_{1}}{2}\right)} r_{w} \frac{1}{\beta \rho} \tau_{\text {Wind }} \tau_{1} \\
& * A^{*}\left\{\mu\left(t-\tau_{2}-\frac{\tau_{1}}{2}\right) b_{0} T\left(t-\tau_{2}-\frac{\tau_{1}}{2}\right)\right. \\
& \left.\quad+A_{\text {Wind }_{\mathrm{n}}}\left(t-\tau_{2}-\frac{\tau_{1}}{2}\right)\right\}
\end{aligned}
$$



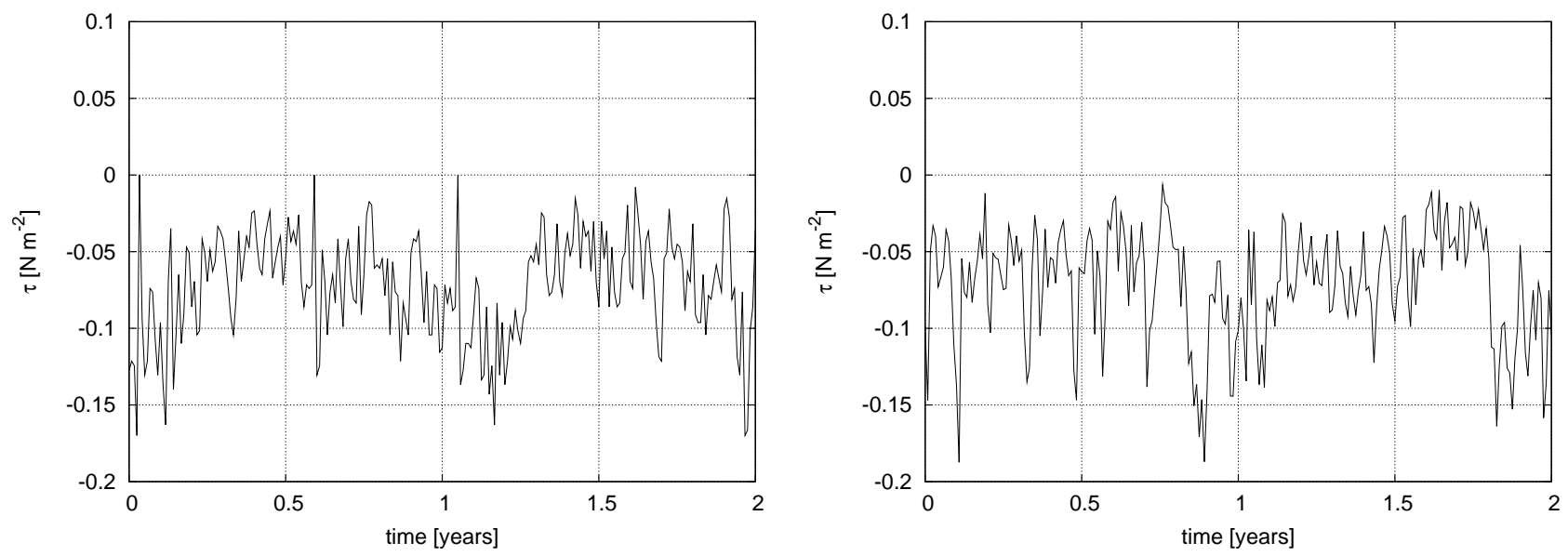

Fig. 1. Windstress: measurement (left) and surrogates (right). The phase and amplitude conservation of lower frequencies including the annual cycle can be seen. The phase of higher frequencies follows a uniform random distribution.

$$
\begin{array}{r}
\left.+e^{-\epsilon_{m} \frac{\tau_{2}}{2} \frac{\tau_{\text {Wind } \tau_{2}}}{\rho C_{o}}\{\mu(t-} \frac{\tau_{2}}{2}\right) b_{0} T\left(t-\frac{\tau_{2}}{2}\right) \\
\left.+A_{\text {Wind }_{\mathrm{e}}}\left(t-\frac{\tau_{2}}{2}\right)\right\}
\end{array}
$$

To study the influence of the additional forcing terms on the model behavior in general, we performed model runs with simple concepts of additional forcing (constant-, gaussian-, sinusoidal-gaussian forcing). From these model realizations, we calculated the corresponding spectra, attractors and amplitude-period relations (For details see Appendix B). By comparison of spectra and attractors of the stochastic runs with those of the standard deterministic model, we obtained the following results:

Forcing with different constants results in different model attractors. One can interpret additional noise as continued change to these constants, thus causing jumps between the attractors. Therefore, the reconstructed attractor under the influence of noise is similar to the undisturbed chaotic attractor (see Fig. 4) where the system jumps between different resonance frequencies (Tziperman, 1995).

The complexity of the results, i.e. the number of different reached attractors, depends on the amplitude and mean value of the noise forcing. Here, a moderate mean value added to a low amplitude noise has the same effect as a zero mean noise with a considerably larger amplitude. Zero mean noise with small amplitude has only a negligible effect.

Since the model is driven by seasonal modulation of the ocean-atmosphere coupling, the impact of the additional forcing also varies throughout the year. Consequently, the existence and the right phase relation of annual variability in the additional forcing have proven to be of great importance to increase model variability.

In order to provide a realistic forcing, we constructed nonzero mean windstress surrogates from windspeed data measured by central pacific buoys in the following way: The spectral band representing the annual (+/ - a half year) variability has been kept to conserve the phase and shape of the annual cycle of the real data. Lower frequencies have been erased to ensure that ENSO variability present in the wind data does not affect our analysis and thus avoiding circular reasoning. To account for stochastic variability, we randomized the phases of high frequency contributions. These surrogates are added to the model's internal windstress, with an additional amplitude of less than 10 percent (for further details, see Appendix A).

The correct construction of surrogates proved to be crucial for the success of this method. In this surrogate driven model (SGT), the generation of ocean waves is no longer bound strictly to the internal state of the oscillator, especially the slowly changing SST, but is also subject to external influences. The latter have a stochastic component but are not entirely random (see Fig. 1). This corresponds to findings of Eisenman and Tziperman (2005).

\section{The noise driven oscillator}

The quality of the model output is evaluated against the SST reconstruction done by Kaplan (1998). This global data set covers the years 1856-1991. From the time series, integrated over the NINO3 region of this data set, we derived the power spectrum and a simple amplitude-period relation (APR). The derivation of these diagnostics are explained in Appendix B.

\subsection{Spectrum}

The GT undergoes the quasiperiodic route to chaos (Eccles and Tziperman, 2004). For a detailed explanation of this route, see Tziperman (1995). Depending on the strength of the seasonal forcing, one can distinguish three regimes of the 

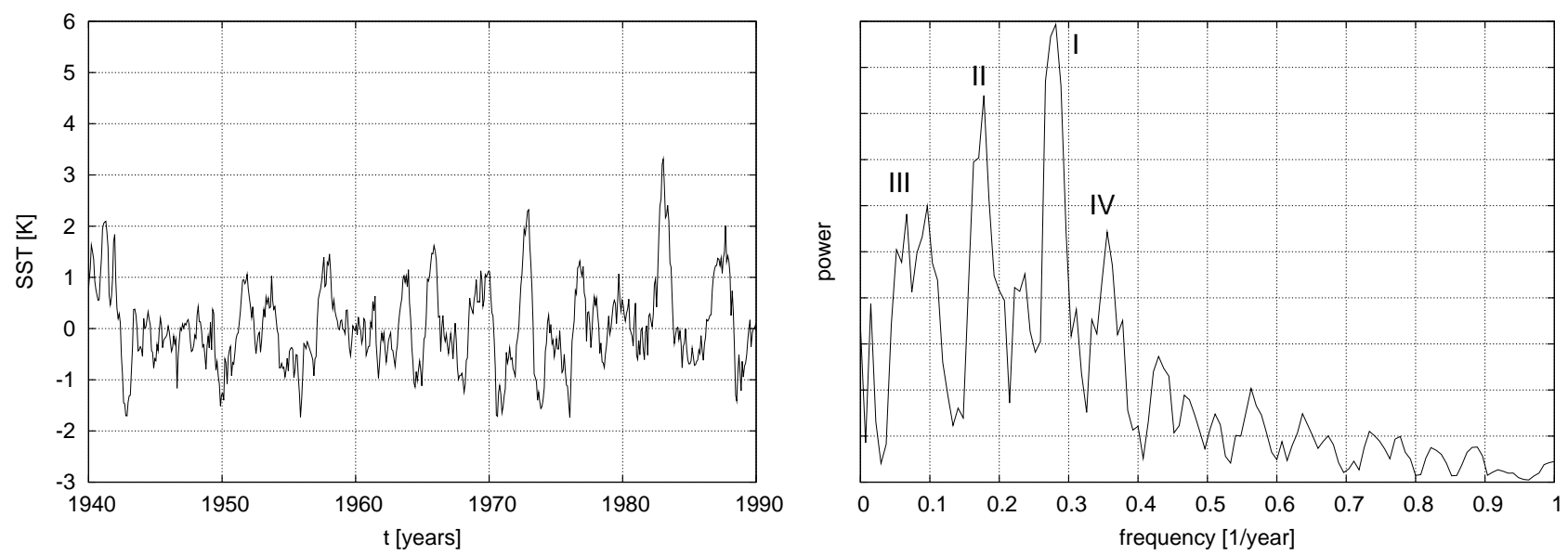

Fig. 2. SST (left) and power spectrum (right) of the Kaplan NINO3 reconstruction. In the time series, the multiscale variability of ENSO can be seen. This results in a broad spectrum with at least four characteristic peaks of the following periods: I: 3.6 years; II: 5.8 years; III: 10-20 years; VI: 2.8 years.

GT (periodic/quasiperiodic, modelock, chaotic). It is evident that all regimes lack the observed ENSO variability when one compares GT spectra (see left panel of Fig. 3) with observations (see Fig. 2).

All GT regimes are able to reproduce the main El Niño peak but fail to produce the spectral bandwidth of the Kaplan reconstruction. It is not surprising that the broadest spectrum and therefore the most variability is produced by the chaotic regime.

The addition of stochastic forcing with power in the frequency band of one per year or higher as defined in Sect. 2.2 increases model variability in general (see right panel of Fig. 3). This especially affects the El Niño spectral main band, i.e. frequencies of interannual variability. Here, additional oscillations are excited and the lower frequencies gain more power in general. This is very astonishing since the additional forcing strength is only a few percent of model generated windstress and has no power in the affected ENSO spectral band at all. Estimation of correlation dimensions (not shown) revealed that the dynamics of a regime is not altered by our additional forcing. It is of interest that the periodic regime is not able to show much variability even under noise forcing. Therefore, we regard the periodic regime as less able to reflect natural conditions.

By making small changes to the parameters we were able to find a regime (hereafter called the spectral optimized regime, SOR) which is well able to reproduce the Kaplan spectrum and the variability of the SST. The similarity is especially remarkable when keeping the conceptual nature of the model in mind (Fig. 5). The parameterization of this regime has a stronger seasonal cycle and therefore has a stronger nonlinearity than the modelock model runs. The SOR may therefore show weakly chaotic behavior.

\subsection{Stability}

Many parameters of the GT are idealized parameterizations of complex processes that are subject to slow and rapid changes, e.g. it is unlikely that the latitude of Rossby wave propagation is constant as represented by the second model stripe. Fluctuations of this latitude result in a change of propagation speed and reflection coefficient. A conceptual model naturally disregards certain physical mechanisms. However, it should react robustly to reasonable parameter variations. Therefore, we did a pragmatic stability analysis of the SGT and the deterministic GT to study the robustness of these models to such parameter variations. The aim was to find the parameter regime that produces realistic variability comparable to the Kaplan data and simultaneously behaves robustly under natural conditions, i.e. small parameter changes. To this end, we slightly altered the parameters of Table 1 and utilized a simple measure $(V)$ to evaluate resulting spectral changes:

$V_{ \pm}(p, \delta p)=\frac{1}{N} \sum_{n=1}^{N} \frac{\left(s_{n}(p)-s_{n}(p \pm \delta p)\right)^{2}}{s_{n}(p)^{2}}$

where $s_{n}(p)$ is the spectral power of the $n$th frequency produced by parameter set $p$. The spectral power $s_{n}(p \pm \delta p)$ is produced by a slightly different parameter set.

We applied variations of a few percent to one parameter at a time. The calculation of $V$ was repeated for the four regimes (periodic, modelock, SOR, chaotic). As expected, the periodic regime results to be the most stable while the spectrum of the chaotic regime turns out to react very sensitively even to minor parameter changes. In the latter regime, a Kaplan-like spectrum is only reproduced for an unrealistically strongly confined parameter region (see Table 2 ). 

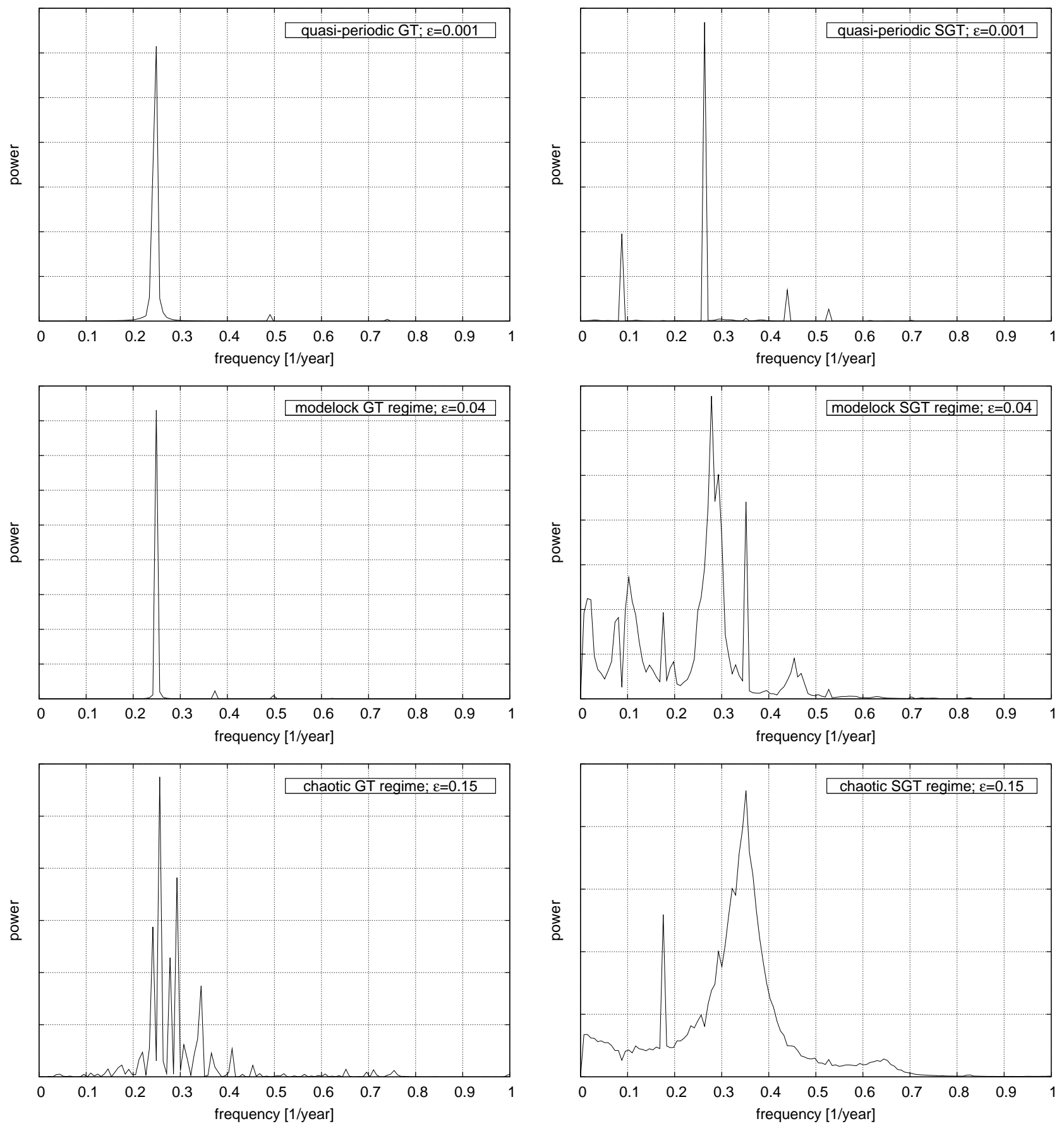

Fig. 3. Quasiperiodic route to chaos: Power spectrum of the deterministic model (GT, left) and the stochastically forced model (SGT, right). The weakly nonlinear deterministic regimes exhibit a single oscillation at the ENSO main frequency. The deterministic chaotic regime generates a small spectral band around that frequency. In all regimes, the applied stochastic forcing broadens the spectrum and excites strong additional oscillations.

From this point of view, the chaotic regime, despite showing reasonable variability, seems not to be suitable to represent the real ENSO system. On the contrary, under parameter changes of $5-10 \%$ the SOR shows a broad spec- trum with characteristic peaks and an El Niño-like time series. In Fig. 6 a sample comparison between the SOR and the chaotic regime of the SGT is shown. Each graph consists of three spectra that correspond to slightly different 

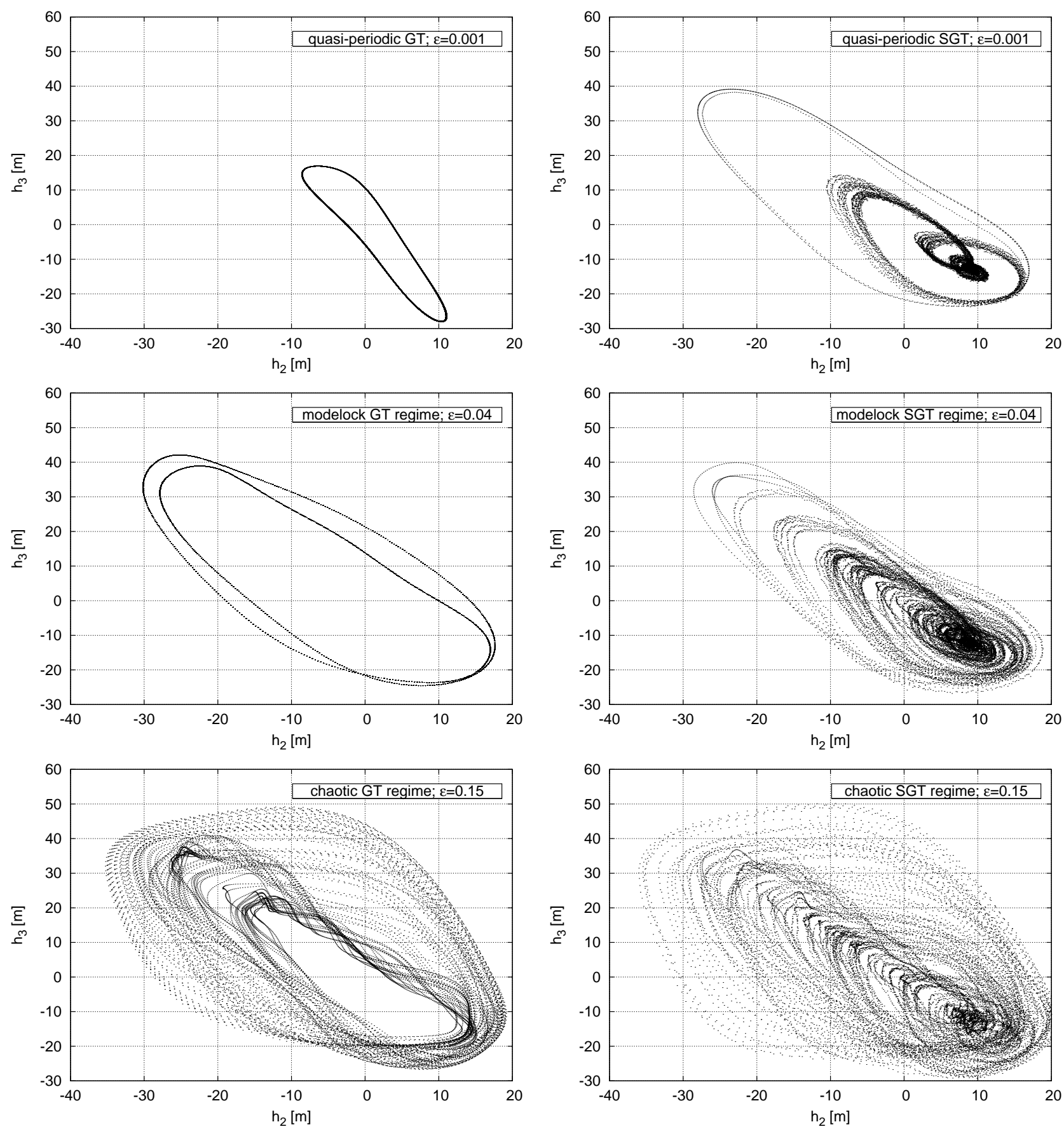

Fig. 4. Quasiperiodic route to chaos: Attractor of the deterministic model (GT, left) and the stochastically forced model (SGT, right). The route to chaos results in a growing attractor of increasing complexity. The applied stochastic forcing also results in an increased complexity.

parameter-sets $(-\delta p ; \pm 0 ;+\delta p)$. The varied parameters in this figure are: ocean-damping $\left(\epsilon_{m}\right)$, western wave reflection coefficient $\left(r_{w}\right)$ and mean upwelling strength $(\bar{\omega})$. It becomes evident that the SOR spectrum is robust under the applied parameter variations. In some parameters, the spectrum of the chaotic regime is equally or even more robust (see end of Ta- ble 2) than the SOR, but in general the chaotic regime tends to change dramatically under slight parameter variations. 

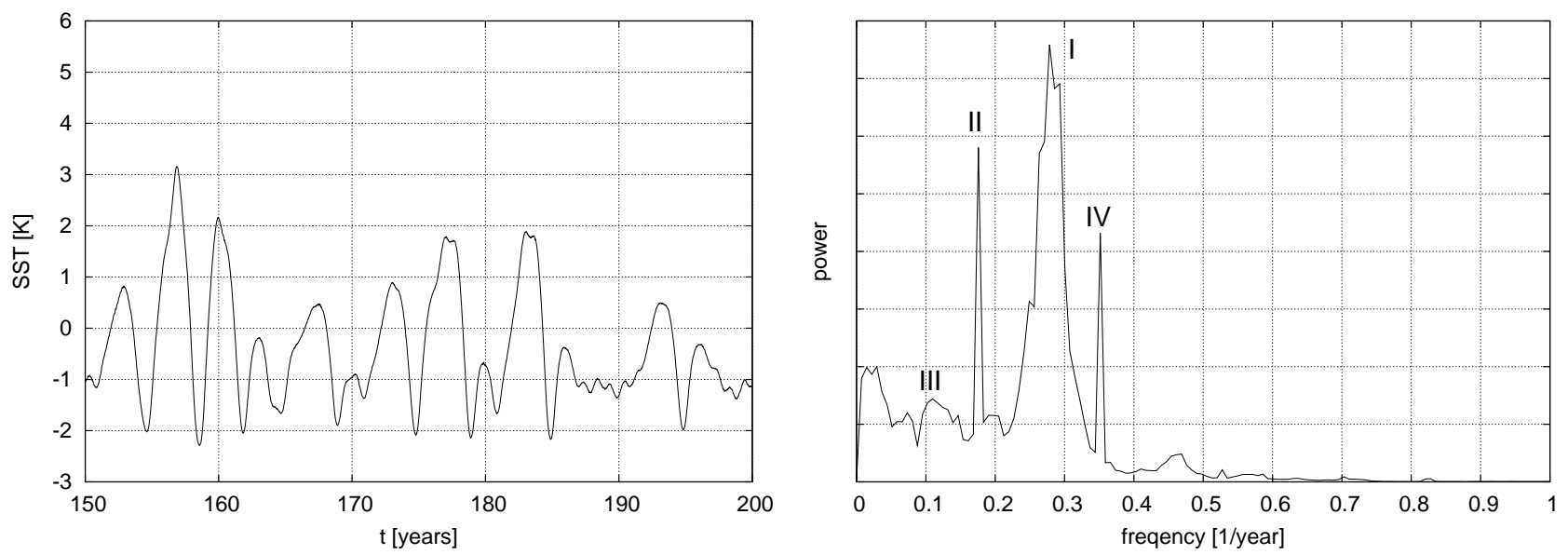

Fig. 5. SST (left) and power spectrum (right) of the spectral optimized regime (SOR) of the stochastic model ( $\varepsilon=0.7)$. The model exhibits El Niños of various amplitude and period similar to the Kaplan data (see Fig. 2). This results in a model spectrum with recognizable ENSO main peaks (I-IV). Obviously the high frequency variability is not captured by the model.

\subsection{Amplitude-period relation}

It is still unclear whether a strong El Niño has a longer or shorter duration compared to a weaker one, or even if El Niño period and amplitude are rather independent of each other. Eccles and Tziperman (2004) obtained the following results in their study of the GT model: For the chaotic regime, they inferred an inversely proportional relation, i.e. the stronger the El Niño, the shorter its duration. For the modelock and periodic regime, however, they found a proportional relation instead, i.e. stronger El Niños last longer than weaker ones. Since the latter regimes have an constant intrinsic period, the investigations had to be rather indirect. By parameter variations, period changes were induced. An additional approach to this question is possible for models with noise (see Appendix B): As the stochastic forcing of the SGT results in amplitude and period variations in all regimes, we could investigate the APR directly even in the weakly nonlinear regimes. The left panel in Fig. 7 shows the results of the deterministic GT, the right panel those of the stochastic SGT.

For the periodic and modelock regime of the GT, our approach revealed a one and a two point relation (cf. corresponding attractors shown in Fig. 4). Our result for the chaotic regime confirms the inversely proportional relation found by Eccles and Tziperman (2004).

The periodic stochastic regime shows a obvious proportional relation of period and amplitude. While in the stochastic modelock case a proportional tendency is still recognizable, no obvoius APR seems to exist in the stochastically disturbed chaotic case. Further increase of the coupling to the seasonal cycle results in an inversely proportional relation (not plotted). Hence, both findings of Eccles and Tziperman (2004) hold for the stochastically forced model too.
Table 2. Regime sensitivity to variations in parameter-set

\begin{tabular}{ccc}
\hline Parameter & SOR $\left(V_{-}+V_{+}\right)$ & Chaotic regime $\left(V_{-}+V_{+}\right)$ \\
\hline$r_{w} \pm 1 \%$ & 0.48 & 394.41 \\
$r_{e} \pm 1 \%$ & 0.23 & 11.79 \\
$\epsilon_{m} \pm 5 \%$ & 0.22 & 96.71 \\
$\epsilon_{t} \pm 5 \%$ & 0.22 & 20.68 \\
$\bar{\omega} \pm 1 \%$ & 0.20 & 36.34 \\
$H 1 \pm 1 \%$ & 0.28 & 31.96 \\
$\rho \pm 1 \%$ & 1.13 & 1.17 \\
$L \pm 1 \%$ & 0.38 & 0.55 \\
$b_{0} \pm 1 \%$ & 0.56 & 0.29 \\
\hline
\end{tabular}

The fundamental difference between the model APR of strong (chaotic) and weakly nonlinear (quasiperiodic) regimes can be utilized for a comparison with measured data: For the NINO3 region SST data, we obtained a rather proportional relation (see Fig. 8). This result is neither matched by the chaotic regime nor by the SOR in deterministic or stochastic model runs. On the other hand, the stochastically forced model runs show more conformity with the observations. Within the SGT runs, the modelock and the modelock/weakly chaotic SOR resemble the observed relation best.

\section{Summary and conclusions}

We investigated the influence of additional surrogate forcing on the variability of a conceptual ENSO model. The forcing noise shares the power spectrum as well as the shape and 

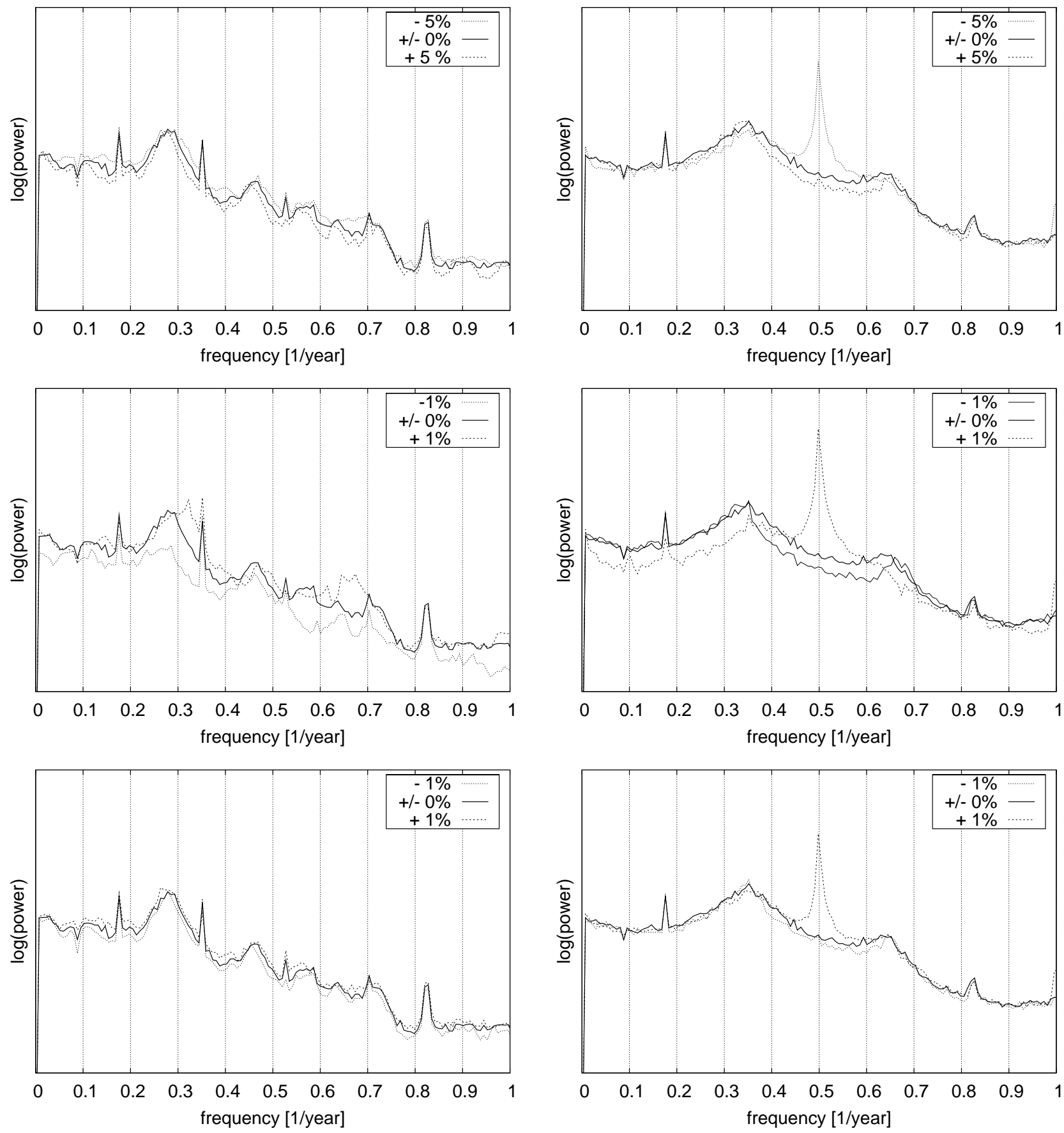

Fig. 6. Power spectrum variations of the spectral optimized regime (SOR, left) and of the chaotic regime (right) of the stochastically forced model. Upper panel: Variation of ocean-damping $\left(\epsilon_{m}\right)$. Middle panel: Variation of western wave reflection coefficient $\left(r_{w}\right)$. Lower panel: Variation of mean upwelling strength $(\bar{\omega})$.

phase of the annual cycle with TOGA/TAO windspeed data. The quality of the model runs was evaluated in the following way: We compared the model spectra and amplitude-period relation (APR) to the corresponding counterparts of observed NINO3 region SST data. As complex physical processes are represented by simple parameters in conceptual models, these parameters are assumed to be subject to considerable fluctuations. Thus, as a further criterion, we investigate the model's ability to produce a robust and realistic spectrum under reasonable parameter changes. A measure of robustness was introduced for this purpose. 

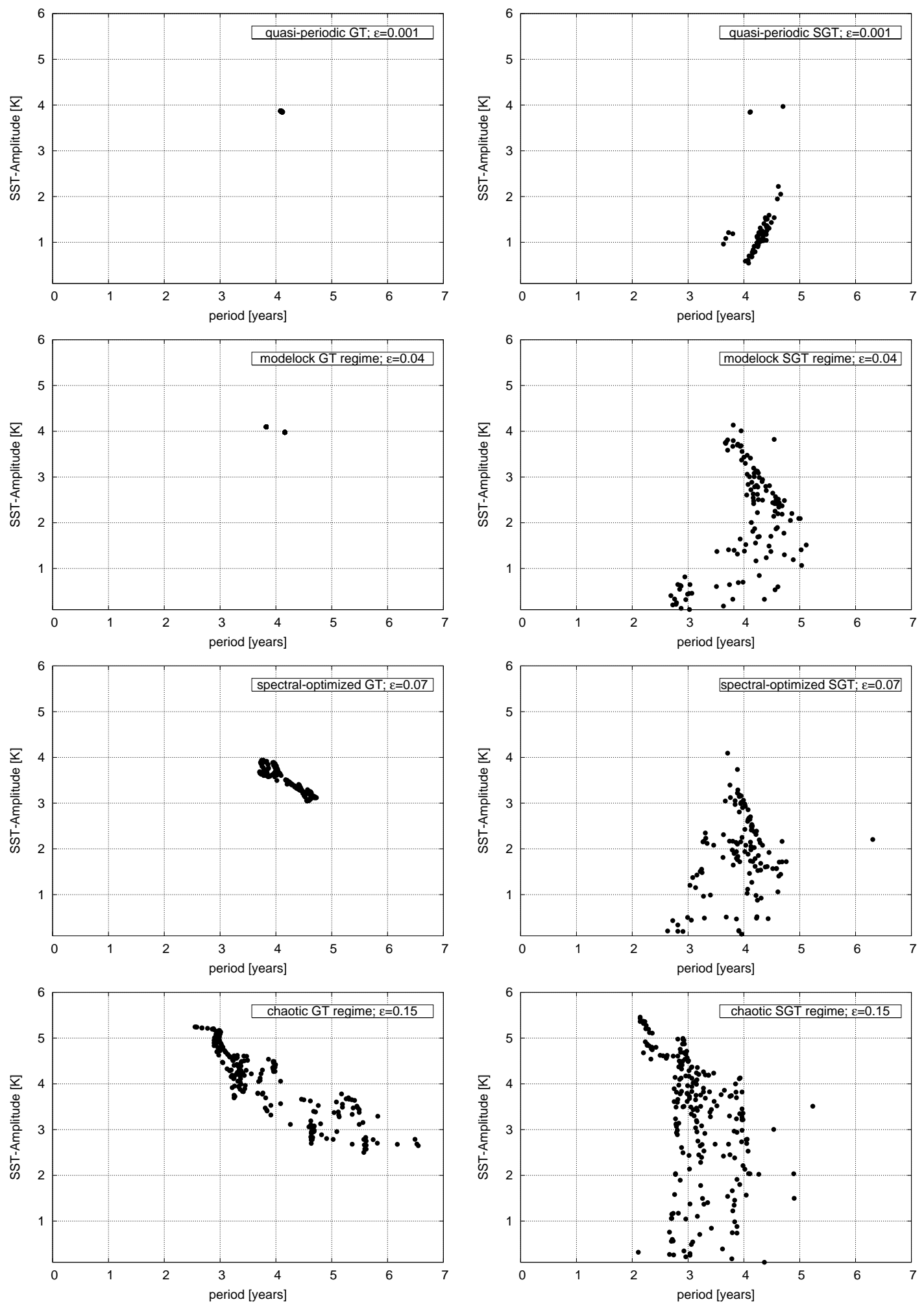

Fig. 7. Amplitude-period relation of GT (left) and SGT (right). While no APR could be derived from the deterministic regimes with weak nonlinearity, the deterministic chaotic regime shows an inversely proportional relation. The stochastically forced regimes show a clearly proportional APR which is most distinct in the periodic case. With increasing nonlinearity, a growing influence of an inversely proportional relation is visible. All model APRs cover only periods longer than 2 years. 


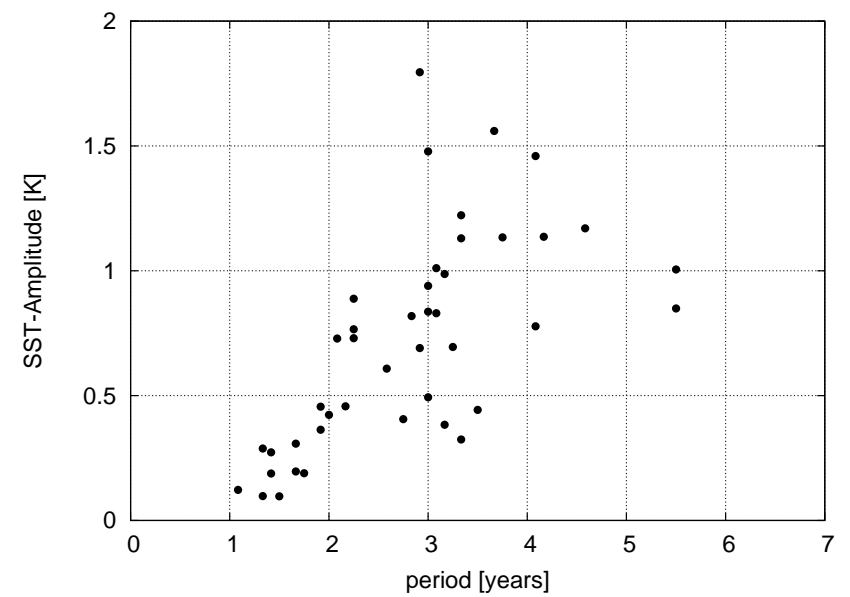

Fig. 8. Amplitude-period relation of the Kaplan NINO3 reconstruction. A rather proportional relation is visible.

The results of our study were the following:

The stochastic and the deterministic periodic regime are very stable but show insufficient variability. Thus, the observed spectrum and APR are reproduced only rudimentarily.

While the deterministic modelock regime also lacks complexity, the stochastic forcing leads to a drastically increased variability. The spectrum and the APR match the observations well. The model output was found to be robust under realistic parameter variations.

The deterministic chaotic regime shows a rather complex behavior but still does not match the bandwidth of the Kaplan spectrum. Adding the stochastic forcing results in a further increment of variability and therefore spectral bandwidth. Nonetheless, the resemblance with the observations is easily destroyed by slight parameter changes. Furthermore, the deterministic chaotic APR and its stochastic counterpart show a strong inversely proportional tendency that does not fit the observations at all.

To summarize, the spectrum, APR and stability analysis favor regimes with medium nonlinearity and additional stochastic forcing. In particular, a modelock/weakly chaotic regime disturbed by surrogate data (SOR) representing tropical pacific surface winds well matching our criteria could be found: This regime resembles the spectrum of NINO3 region SST measurement data, i.e. the variability within the ENSO spectral band and the four ENSO main spectral peaks were reproduced. Additionally, the APR is consistent with the observed one. Finally, this regime turned out to be stable under reasonable parameter changes.

The inability of all deterministic regimes to express observed ENSO variability, and the result that additional forcing raises the spectral power within the ENSO main band (2-7 years), suggest that atmospheric noise plays a significant role for the ENSO dynamics. This external influence includes high frequency weather noise as well as low frequency windstress variations that interact with the seasonal cycle presented by ocean-atmosphere coupling. However, all these results have to be interpreted in the light of the conceptual origin of this model. Thus, it would be interesting to repeat this study with more complex models.

\section{Appendix A}

\section{Construction of the surrogates}

The construction of the surrogates turned out to be a crucial part of this work. The construction is based upon zonal windspeed data taken from the TOGA/TAO buoy array. After removal of the mean value, we applied a Fourier transformation. In the frequency domain, we performed two essential operations. First, we used a low pass filter to erase every period longer than one and a half years from the spectrum. This operation eliminates the main El Niño time scales from the time series. Second, we added a uniform distributed phase to all oscillations with periods shorter than 100 days. Note that this second procedure does not alter the power spectrum of the data set. After inverse Fourier transformation and addition of the mean value, we now have a new windspeed time series. This time series has exactly the same power spectrum as the original one except for the deleted low frequencies. Furthermore, the oscillations between 1.5 years and 100 days keep their original phase relations to interact with the seasonal cycle of the model in a natural way. Finally, the phases of quick oscillations faster than 100 days are disturbed randomly to represent the stochastic component of the atmosphere. A simple drag relation turns this time series into windstress.

In order to pay tribute to the two ocean stripes of the GT, we derived our surrogates from two different locations of the central pacific $(0 \mathrm{~N} 170 \mathrm{~W}, 8 \mathrm{~N} 170 \mathrm{~W})$. In this way, we could force the two stripes independently of each other with characteristic time series. Model runs brought best results with an amplitude of the additional forcing that corresponds to $2.8 \%$ (respectively $9.8 \%$ ) of the negative (respectively positive) undisturbed, internal model windstress in the equatorial stripe. In the off-equatorial stripe, the forcing matches $0.8 \%$ (respectively 2.8\%) of the model-generated windstress. This corresponds only to a few thousandth of observed windstress and is because the GT generates relatively weak windstress compared to observations.

\section{Appendix B}

\section{Diagnostics}

While the Kaplan power spectrum is constructed in the usual manner. To derive the APR, we determined all local extrema that were at least 19 months apart, and locally fitted parabola 
to the extrema. The amplitude of an El Niño was taken to be the mean of a maximum and the two neighboring minima. We defined the corresponding period as the time span between those enclosing minima.

The power spectra of the model are the means over five thousand time windows (each 140 years long and far away from the models spin up). Derivation of the model's APR was done by taking duration and maximum SST of every attractor cycle. The attractor used for this purpose was built by taking the three terms of Eq. (4) as components of a three dimensional vector. This reconstruction is isomorphic to attractors gained by the embedding of model SST but period dependent folding problems are avoided.

Acknowledgements. We would like to thank our colleague B. Blasius for enlightening discussions and J. Ong for his support.

Edited by: M. Thiel

Reviewed by: two referees

\section{References}

Bjerknes, J.: Atmospheric teleconnections from the equatorial Pacific, Mon. Wea. Rev., 97, 163-172, 1969.

Boulanger, J.-P., Menkes, C., and Lengaigne, M.: Role of high- and low-frequency winds and wave reflection in the onset, growth and termination of the 1997-1998 El Niño, Clim. Dyn., 22, 267280, 2004.

Eccles, F. and Tziperman, E.: Nonlinar effects on ENSO's period, J. Atmos. Sci., 61, 474-482, 2004.
Eisenman, I., Yu, L., and Tziperman, E.: Westerly Wind Bursts: ENSO's tail rather than the dog?, J. Climate, 18, 5224-5238, 2005.

Fedorov, A. V., Harper, S. L., Philander, S. G., Winter, B., and Wittenberg, A.: How Predictable is El Niño?, Bull. Amer. Meteorol. Soc., 84, 911-919, 2003.

Galanti, E. and Tziperman, E.: ENSO's phase locking to the Seasonal Cycle in the Fast-SST, Fast-Wave, and Mixed-Mode Regimes, J. Atmos. Sci., 57, 2936-2950, 2000.

Kaplan, A., Cane, M. A., Kushnir, Y., Clement, A. C., Blumenthal, M. B., and Rajagopalan, B.: Analyses of global sea surface temperature 1856-1991, J. Geophys. Res., 103, 18 567-18 589,1998.

Kessler, W. S.: Is ENSO a cycle or a series of events?, Geophys. Res. Lett., 29(23), 2125-2128, 2002.

Lengaigne, M., Guilyardi, E., Boulanger, J. P., Menkes, C., Delecluse, P., Innes, P., Cole, J., and Slingo, J.: Triggering of El Niño by westerly wind events in a coupled general circulation model, Climate Dyn., 23(6), 601-620, 2004.

Stone, L., Saparin P. I., Huppert, A., and Price, C.: El Niño chaos: the role of noise and stochastic resonance on the ENSO cycle, Geophys. Res. Lett., 25(2), 175-178, 1998.

Thompson, C. J. and Battisti, D. S.: A linear stochastic dynamical model of ENSO. Part II: Analysis, J. Climate, 14, 445-466, 2001.

Timmermann, A. and Jin, F. F.: A Nonlinear Theory for El Niño Bursting, J. Atmos. Sci., 60, 152-165, 2003.

Tziperman, E., Stone, L., Cane, M. A., and Jarosh, H.: El Niño Chaos: Overlapping of Resonances Between the seasonal Cycle and the Pacific Ocean-Atmosphere Oscillator, Science, 264, 72$74,1994$.

Tziperman, E., Cane, M. A., and Zebiak, S. E.: Irregularity and Locking to the Seasonal Cycle in an ENSO Prediction Model as Explained by the Quasi-Periodicity Route to Chaos, J. Atmos. Sci., 52(3), 293-306, 1995. 